\title{
Biodiversidad en cifras: avances en el conocimiento de especies en Costa Rica
}

\author{
Biodiversity in Numbers: Update on Species Knowledge in Costa Rica \\ Tania Bermúdez Rojas* \\ Vilma Obando Acuña*
}

DOl: $10.22458 /$ rb.v32i2.3899

Recibido - Received: 29/09/2021 / Corregido - Revised: 18/10/2021 / Aceptado - Accepted: 04/11/2021

\section{RESUMEN}

Para conservar y utilizar sosteniblemente la biodiversidad es fundamental conocerla, por ello se presentan los datos en cifras de algunos grupos taxonómicos de Costa Rica. La información fue recopilada, analizada y sistematizada en talleres, consulta a especialistas y revisión de publicaciones. Se presenta la información de forma integrada y sencilla, comparando las cifras del 2014 con el 2019 como actualización de los siguientes grupos taxonómicos: insectos, hongos, líquenes, plantas, peces, anfibios, reptiles, aves y mamíferos. Además, se mencionan los principales vacíos de información. Entre los principales resultados observamos que para el 2019 aumentó el número en la mayoría de los grupos de vertebrados, siendo los peces los de mayor incremento; para anfibios, reptiles, aves y mamíferos, el aumento fue menor debido a las revisiones taxonómicas que permitió el ingreso, pero también la eliminación de algunos registros. El número de insectos, así como los artrópodos están en revisión. El número de plantas y hongos disminuyó, por la rectificación taxonómica de los dos grupos. Sin embargo, separando hongos de líquenes, este último presenta un incremento significativo. La información generada es fundamental para comenzar a generar datos serios, confiables y actualizados sobre nuestra biodiversidad.

Palabras clave: diversidad biológica; conservación; datos de especies; grupos taxonómicos; vacíos de información.

\section{ABSTRACT}

To preserve and use biodiversity in a sustainable way, it is fundamental to know it. This is the reason why data is presented for some taxonomic groups of Costa Rica. Information was collected, analyzed, and systematized in workshops, specialist consultations and publication reviews. Information is presented in an integrated and simple way, comparing numbers between 2014 and 2019 as an upgrade for the following taxonomic groups: insects, fungi, lichen, plants, fish, amphibians, reptiles, birds, and mammals. Furthermore, information gaps are mentioned. Among the main results, for 2019 the number of most vertebrate groups had risen, with fish as the one which had the biggest increment. For amphibians, reptiles, birds, and mammals, the increment was lower because of the taxonomic revisions that allowed the entry but also the elimination of some registrations. The number of insects as well as arthropods are in revision. The number of plant and fungi decreased because of taxonomic rectification for both groups. However, separating fungi from lichen, the last one had a significative increment. Information generated is fundamental to begin a baseline of serious, reliable, and up to date data about our biodiversity.

Key words: biological diversity; conservation; species data; taxonomic groups; information gaps.

* Laboratorio de Biología Tropical. Escuela de Ciencias Biológicas. Universidad Nacional, Heredia, Costa Rica. tania.bermudez.rojas@una.ac.cr ID: https://orcid.org/0000-0002-7566-9521

** Escuela de Ciencias Biológicas. Universidad Nacional, Heredia, Costa Rica. vilma.obando.acuña@una.ac.cr ID: https://orcid.org/0000-0002-8800-1360 


\section{Introducción}

Costa Rica ha sido un líder mundial en los temas ambientales y de conservación de la biodiversidad, particularmente desde finales de los años 80 participó activamente antes, durante y después de la Cumbre de la Tierra y sigue siendo referente en el tema. Su enfoque innovador y pionero, especialmente en el marco de salvar, conocer y usar sosteniblemente la biodiversidad, ha sido la marca nacional a la cual se encadenan numerosas actividades productivas como el turismo.

Uno de los primeros pasos para establecer lineamientos para conservación y utilización sostenible de la biodiversidad parte de conocerla. Los esfuerzos nacionales para entender y saber sobre la riqueza biológica vienen desde el siglo 19; han sido diversos y numerosos y desde diferentes sectores, especialmente el académico. Integrar la información y transferirla de la mejor forma posible a todo aquel que la requiera sigue siendo un reto país.

En el año 1992, se realizó el primer Estudio Nacional de Biodiversidad (MIRENEM, Museo Nacional, INBio, 1992), el cual no fue publicado oficialmente por falta de financiamiento; sin embargo, sentó la base para contar con un número inicial de especies de varios grupos taxonómicos y su estado de conservación, entre ellos los mejor conocidos: plantas y vertebrados.

A partir de la información, y como parte del proceso de formulación de la Estrategia Nacional de Biodiversidad y el I Informe de País al Convenio sobre la Diversidad Biológica (CDB), el Instituto Nacional de Biodiversidad (INBio) en coordinación estrecha con el Sistema Nacional de Áreas de Conservación (SINAC), como ente rector en el tema, empiezan en el año 1998 la actualización de datos de biodiversidad, mediante talleres, consulta a especialistas, grupos focales y revisión de publicaciones. Los datos generales se divulgaron en ese momento y posteriormente en forma periódica por diferentes medios, hasta el año 2014.

La información no se volvió a actualizar hasta el año 2019, cuando la Escuela de Ciencias Biológicas (ECB) de la Universidad Nacional asumió el reto, con la ejecución de un proyecto denominado: Biodiversidad en Cifras, en coordinación estrecha primero con el SINAC, luego con la Comisión Nacional de Gestión de la Biodiversidad (CONAGEBIO) y paralelamente con el Museo Nacional, así como con la Comisión Interinstitucional para la Gestión del Conocimiento y la Información sobre Biodiversidad (CIGECIB).

Uno de los objetivos principales que pretende el proyecto Biodiversidad en Cifras es la divulgación de la información sobre la riqueza biológica de forma integral y sencilla, que se transfiera por diferentes medios como aporte a su conservación y uso sostenible, marca país y ejemplo mundial.

\section{Metodología}

La actualización de datos de biodiversidad se realizó mediante talleres, consulta a especialistas, grupos focales y revisión de publicaciones. Hasta el momento se han realizado cinco talleres de expertos con la participación de 55 personas, dos reuniones con especialistas de grupos claves (insectos acuáticos y nematodos), revisión de literatura, información brindada en el Simposio de Botánica del Museo Nacional realizado en octubre 2019 y consulta a las bases de datos existentes. 
El resultado a la fecha es un primer acercamiento a la integración de información actualizada sobre el número de especies del país, faltando aún la actualización de grupos muy abundantes, pero poco estudiados, tal como los insectos y los microorganismos, de los cuales solo se tienen datos integrados al 2014 para el primero y de 1992 para el segundo.

\section{Actualización de datos \\ El mundo y Costa Rica. \\ Datos cambiantes}

La estimación sobre el número de especies conocidas (descritas) en el planeta es de aproximadamente 1900000 con un esperado cercano a los 8700000 , faltan así $78 \%$ de especies por ser descritas o descubiertas (Mora et al., 2011). Los datos de especies descritas y esperadas en el mundo varían según la fuente y no todos los países reportan la información.

Un estudio publicado en setiembre de 2017, de Larsen et al., habla de cerca de 1500000 especies descritas y de entre 1 a 6 billones de especies esperadas en la Tierra; lo anterior gracias a profundos análisis que realizaron tomando en cuenta numerosos grupos de organismos no incluidos en estudios previos (basados principalmente en insectos), como bacterias y nematodos. En contraste con otros estudios, los autores publican que la biodiversidad global es dominada por bacterias (entre 70 y $90 \%$, aproximadamente) y los insectos son solamente uno de los muchos grupos hiperdiversos.

En Costa Rica, según los datos de 1992, las bacterias representan tan solo $5 \%$ de las especies esperadas (figura 1), lo que a la luz de los datos de 2017 de Larsen et al. y los resultados del Taller de Microorganismos realizado en el marco del proyecto, el universo no solo por descubrir, sino por integrar, es de enorme magnitud en esos grupos.

Para 1992, el número total esperado de especies para el país se calculó en aproximadamente 500000 , donde $71 \%$ se concentra en insectos (figura1). El dato ha sido considerado desde entonces como un número subestimado; hoy día (2021) se habla de que el número podría al menos duplicarse, máxime con las nuevas técnicas de identificación mediante el empleo del ADN, en las cuales el país ya ha incursionado.

Las tendencias en cuanto a la posible riqueza por grupo en general se mantienen, con la excepción de que los microorganismos podrían estar muy por arriba de lo esperado en 1992, especialmente en relación con bacterias, como se mencionó anteriormente.

\section{Figura 1}

Porcentaje de especies esperadas para Costa Rica al año 2014

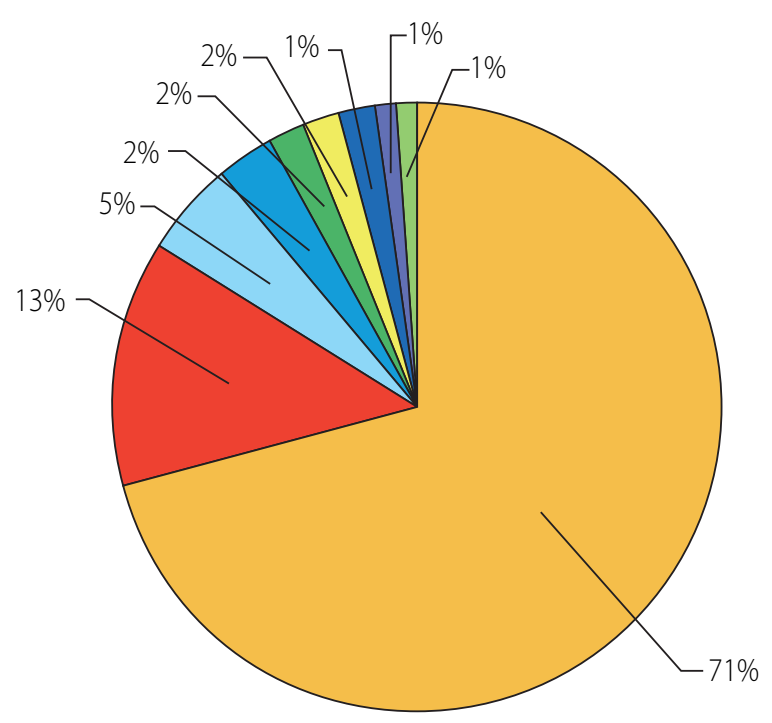

Nota. Basado en el Estudio de Biodiversidad de 1992 (SINAC, INBio, Museo Nacional, 1992). 
Al 2013-2014, el número de especies conocidas en el país era de 94778 incluyendo el grupo de microrganismos (que seguía siendo el número dado en 1992), lo cual equivale a cerca de $4,75 \%$ de la biodiversidad descrita en el mundo (tomando el dato de 1814 156, cuadro 1). El porcentaje cercano a $5 \%$ es el que se ha utilizado por los diferentes sectores desde el año 2002 cuando se divulgó en forma amplia por el INBio-SINAC (Obando, 2002 y 2007), con leves modificaciones en las actualizaciones posteriores hasta el 2014, último año en que el INBio generó datos integrados de nuevas especies. Para ese año, el número de especies para el país subió a 95 157, sumando 381 especies nuevas (datos publicados en el Informe del Estado de la Nación del 2015. CONARE, 2015).

Un nuevo esfuerzo de actualización de números de especies se presentó en 2018, cuando se realizó el VI Informe de País sobre la Implementación del Convenio sobre la Diversidad Biológica (SINAC-MINAE, CONAGEBIO, 2018). El documento informa sobre 121693 de especies descritas. Sin embargo, aunque es de gran importancia, los datos en su mayoría no pueden ser comparables en su totalidad con los del 2014 por la forma en que se desglosaron, y los grupos que se tomaron en cuenta en la sumatoria. Se optó entonces por no tomarlos en cuenta para la comparación con los datos recopilados en los talleres y otras fuentes de información (cuadro 2). Es parte de la tarea por hacer, uniformar datos para que puedan ser comparables.

Para el año 2019, el proyecto Biodiversidad en Cifras actualizó datos de vertebrados y plantas. El número de especies aumentó en la mayoría de los grupos de vertebrados, siendo los peces el que reportó el mayor incremento con 859 especies más con respecto al 2014 (cuadro 2).

En el caso de anfibios, reptiles, aves y mamíferos (figura 2), el aumento fue menor, con un rango de dos a diez nuevas especies; no obstante, se debe aclarar que la actualización incluyó tanto nuevos ingresos como eliminación de otras, debido a revisiones taxonómicas. También se observa una disminución en el número de plantas y hongos por la rectificación y aclaración taxonómica de los dos grupos. Sin embargo, separando hongos de líquenes, este último presenta un incrementó de 63 especies (cuadro 2).

\section{Figura 2}

Grupos taxonómicos ya superados (reptiles y aves) respecto al número de especies esperadas al 2014
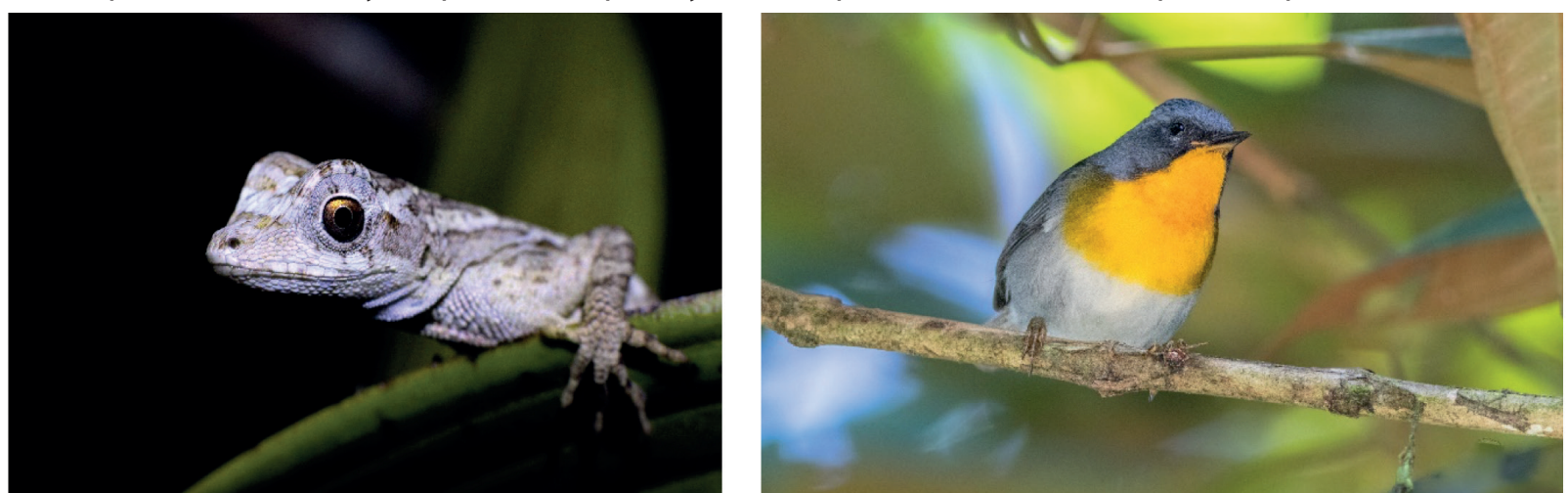

Nota. Especie de reptil Anolis lemurinus (fotografía tomada por Leandro Araya).

Especie de Ave Oreothlypis gutturalis (fotografía tomada por Roberto Vargas). 
Cuadro 1

Datos de número de especies de grupos taxonómicos para Costa Rica. 2013-abril 2014

\begin{tabular}{|c|c|c|c|c|c|}
\hline Grupo & $\begin{array}{c}\mathrm{N}^{\circ} \text { especies } \\
\text { conocidas } \\
\text { en el mundo }\end{array}$ & $\begin{array}{c}\mathrm{N}^{0} \text { de especies } \\
\text { esperadas } \\
\text { para Costa Rica }\end{array}$ & $\begin{array}{c}\mathrm{N}^{\circ} \text { de } \\
\text { especies } \\
\text { conocidas } \\
\text { para Costa } \\
\text { Rica }\end{array}$ & $\begin{array}{c}\text { \% conocido } \\
\text { de lo } \\
\text { esperado para } \\
\text { Costa Rica }\end{array}$ & $\begin{array}{l}\% \text { conocido } \\
\text { en CR de lo } \\
\text { conocido } \\
\text { en el mundo }\end{array}$ \\
\hline Virus & 1500 & 8000 & 125 & 2 & 8 \\
\hline $\begin{array}{l}\text { Monera (bacterias y algunas microalgas, } \\
\text { como fitoplancton) }\end{array}$ & 8276 & 26350 & 213 & 1 & 3 \\
\hline Protozoarios & 40000 & 8000 & 670 & 8 & 2 \\
\hline Algas & 40000 & 4350 & 564 & 13 & 1 \\
\hline $\begin{array}{l}\text { Hongos (líquenes, macrohongos, } \\
\text { microhongos) }\end{array}$ & 80000 & 65000 & 3873 & 6 & 5 \\
\hline Insectos & 950000 & 365000 & 69109 & 19 & 7 \\
\hline Otros invertebrados & 375000 & 17235 & 5898 & 34 & 2 \\
\hline Plantas & 270000 & 12000 & 11535 & 96 & 4 \\
\hline Vertebrados: & 49380 & 3151 & 2786 & 88 & 6 \\
\hline Vertebrados inferiores & 60 & 2 & 1 & 50 & 2 \\
\hline Peces (marinos y continentales) & 19056 & 1600 & 1187 & 74 & 6 \\
\hline Anfibios & 7000 & 215 & 201 & 93 & 3 \\
\hline Reptiles & 8613 & 230 & 239 & Superado & 3 \\
\hline Aves & 9917 & 854 & 912 & Superado & 9 \\
\hline Mamíferos & 4734 & 250 & 249 & 100 & 5 \\
\hline Total & 1814156 & 509086 & 94778 & 19 & 4,7 \\
\hline
\end{tabular}

Nota. Obando, V., Herrera, A. y Ugalde, J. INBio. 2013, a partir de diferentes fuentes nacionales e internacionales. Para vertebrados: Herrera, A y Obando, V. INBio. Abril, 2014.

El número de insectos y artrópodos esperados para el país en general están en revisión, al igual que las especies descritas. Según Avalos (2018), otros artrópodos como los ácaros pueden tener tantas especies como los insectos, lo que resulta en medio millón de especies de artrópodos solamente (Hanson, P., comunicación personal, en Avalos, 2018). Además, las estimaciones recientes de la biodiversidad de la Tierra (Larsen et al., 2017) pueden aumentar los números de otros grupos (bacterias, hongos, nematodos), afectando sustancialmente los números esperados para Costa Rica.

Biocenosis Volumen 32/Número 2/diciembre 2021
Si se mantienen los números esperados para el 2014, con las actualizaciones hechas en algunos grupos al 2019, los porcentajes de especies esperadas y las descritas o conocidas para Costa Rica, comparados con lo esperado y descrito para el mundo, se mantiene muy similar $6 \%$ y $4,9 \%$, respectivamente.

\section{Vacíos de información}

La gran mayoría de las actualizaciones del número de especies a nivel mundial están sesgadas a especies conspicuas (conocidas), abundantes y con amplia distribución geográfica, como plantas y vertebrados (anfibios, 
Cuadro 2

Número de especies conocidas para los años 2014 y 2019

\begin{tabular}{lccc}
\multicolumn{1}{c}{ Grupos } & $\begin{array}{c}\text { Número } \\
\text { conocido 2014 }\end{array}$ & $\begin{array}{c}\text { Número conocido } \\
\mathbf{2 0 1 9}\end{array}$ & $\begin{array}{c}\text { Número estimado } \\
\text { para Costa Rica 2014 } \\
\text { y actualizaciones }\end{array}$ \\
\hline Insectos & 69109 & 69109 & 365000 \\
Hongos (se incluyen macro y microhongos y líquenes) & 3873 & $3063(2)$ & $250000(1)$ \\
\hline Líquenes & 1500 & $1563(3)$ & $2400(3)$ \\
\hline Plantas & 11535 & $9812(3)$ & $10300(3)$ \\
\hline Peces (marinos y continentales) & 1187 & $2046(4)$ & 1600 \\
\hline Anfibios & 201 & $211(5)$ & 215 \\
\hline Reptiles & 239 & $241(5)$ & 230 \\
\hline Aves & 909 & $927(6)$ & 854 \\
\hline Mamíferos & 249 & $251(7)$ & $257(7)$ \\
\hline
\end{tabular}

Notas. (1) Avalos, G. (2018). Número aproximado. (2) Milagro Mata, especialista en hongos, UNED, comunicación personal, 4 de mayo 2020. La UNED está en proceso de realizar un proyecto de actualización de la lista de Hongos para el país en $2020-2021$. (3) Simposio de Botánica 2019 del Museo Nacional, http://www.museocostarica.go.cr/es_cr/simposio-de-bot-nica/index.php?ltemid=75. Con respecto a plantas, los cambios con respecto al 2014 se deben a revisiones taxonómicas. (4) Angulo, et al. (2013), En: Avalos, G. (2018). (5) Taller SINAC-ECB/UNA, 4 de abril de 2019. (6) Taller SINAC-ECB/UNA, 14 de noviembre de 2019 (7) VI Informe de País al Convenio sobre la Diversidad Biológica (SINAC-CONAGEBIO, 2018) reporta 912 sp. La Asociación Ornitológica reporta 923 sp y la Unión Ornitológica 924 para el 2020, cuando se integran las dos listas el total es de 927 especies, por lo tanto, se deja este número mientras se revisan y oficializan las listas de parte del SINAC en el 2021.

reptiles, peces, mamíferos y aves). En Costa Rica, son los grupos mejor conocidos, en donde varios de los números esperados ya fueron superados (cuadro 1 y 2, figura 2). Como se anotó anteriormente, los microrganismos (figura 3), tanto eucariotas como procariotas y los virus presentan un porcentaje de especies descritas sumamente bajo con respecto a lo esperado (Larsen et al. 2017). Lo anterior también ocurre en Costa Rica donde, desde el año 1992, no se ha actualizado el número de especies en estos grupos. Queda el reto de establecer un mecanismo de actualización, unificación y sistematización referente a microorganismos y la necesidad de digitalizar los datos de las colecciones de instituciones como el Instituto Nacional de Innovación y Transferencia en Tecnología, el Instituto Tecnológico de Costa Rica, la Universidad Nacional y la Universidad de Costa Rica.
Figura 3

Grupos con vacíos de información en biodiversidad en cifras

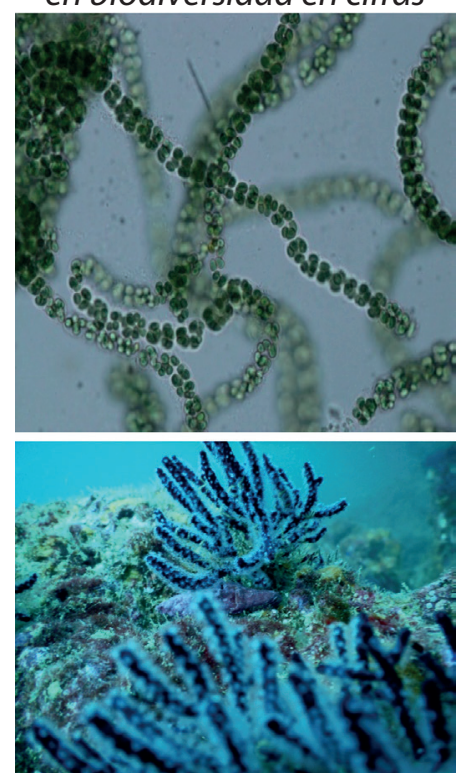

Nota. Microalga, Parthasarathiella prolífica (40X), fotografía del Laboratorio de Biotecnología de Microalgas, UNA. Octocoral, Leptogorgia rigida, fotografía de Karol Ulate Naranjo. 
Otro de los grupos con importantes vacíos de información son las especies marino costeras (figura 3), enfocado principalmente a invertebrados a pesar de que el mar territorial costarricense es 11 veces más grande que el área terrestre. Los únicos datos integrados que posee el país para estos grupos se publicaron en el 2009 en un libro donde se informaba de 6778 especies descritas en diferentes grupos marinos (Wehrtmann I. \& Cortés J., 2009), el cual debe servir de base para actualizar los números. Algunos datos interesantes del libro citado se describen a continuación.

- La publicación establece que la biodiversidad marina conocida del país es de 6778 especies, lo que representa cerca de $3,5 \%$ de la biodiversidad conocida en el mundo.

- De estas especies, 4745 son reportadas para el Pacífico y 2321 para el Caribe. Mientras que en el Pacífico hay cuatro especies diferentes por kilómetro cuadrado de costa, en el Caribe esa cifra es de nueve especies por cada kilómetro cuadrado.

- Ambas costas comparten 288 especies y se establece que existen 85 especies endémicas o que se conocen solo en las aguas de Costa Rica, de estas especies endémicas $41,2 \%$ se encuentra en la Isla del Coco.

Los grupos que tienen especies marinas son sumamente diversos y complejos, la tarea por hacer es conocer las especies en los diversos ecosistemas marino-costeros, de enorme magnitud en el mundo. Una investigación rápida sobre reportes de especies nuevas para grupos poco comunes que no se detallan en el libro de Wehrtmann y Cortés, se muestra en el cuadro 3.
Cuadro 3

Reporte de especies nuevas en grupos con especies marinas poco conocidos al 2019

\begin{tabular}{|c|c|}
\hline $\begin{array}{l}\text { Grupos y cambios } \\
\text { taxonómicos }\end{array}$ & $\begin{array}{c}\text { ¿Se encontró información } \\
\text { de especies nuevas? }\end{array}$ \\
\hline Ctenophora & $\begin{array}{l}\text { No se encontró } \\
\text { ningún dato. }\end{array}$ \\
\hline Entoprocta & $\begin{array}{l}\text { No se encontró } \\
\text { ningún dato. }\end{array}$ \\
\hline Gastrotricha & $\begin{array}{l}\text { No se encontró } \\
\text { ningún dato. }\end{array}$ \\
\hline Rotifera & $\begin{array}{c}\text { Sólo se encuentra literatura } \\
\text { de agua dulce. }\end{array}$ \\
\hline $\begin{array}{l}\text { Cephalorhyncha, Phylum de } \\
\text { Priapulida, ahora es una clase, } \\
\text { antes estaba como Phylum }\end{array}$ & $\begin{array}{l}\text { No se encontró } \\
\text { ningún dato. }\end{array}$ \\
\hline $\begin{array}{l}\text { Cephalorhyncha Phylum de } \\
\text { Kinorhyncha, ahora clase antes } \\
\text { se consideraba Phylum }\end{array}$ & $\begin{array}{c}5 \text { especies reportadas } \\
\text { (Neuhaus \& Blasche, 2006). }\end{array}$ \\
\hline Phoronida & $\begin{array}{c}1 \text { especie reportada } \\
\text { (Dean, H.K. Sibaja-Cordero, } \\
\text { J.A. \& Cortés, J. 2010). }\end{array}$ \\
\hline $\begin{array}{l}\text { Phylum Hemichordata de la } \\
\text { Clase Enteropneusta. Gusano } \\
\text { bellota sin identificar }\end{array}$ & $\begin{array}{l}1 \text { reporte con individuo } \\
\text { No identificado } \\
\text { (Vargas et al 2016). }\end{array}$ \\
\hline
\end{tabular}

Nota. Elaborado por Karol Ulate Naranjo, profesora e investigadora de la ECB-UNA. 26 de julio 2019. Comunicación personal.

\section{El propósito de conocer nuestra biodiversidad}

El conocer el número de especies que cohabitan en nuestro territorio es fundamental para la conservación, manejo y gestión de la biodiversidad, así como para los servicios que provee para el bienestar humano (salvar y usar sosteniblemente), donde la soberanía alimentaria, la salud y el turismo son algunos ejemplos de actividades que dependen de la buena gestión que realice el país de su riqueza biológica. Se tiene la responsabilidad de informar al mundo sobre su avance en el cumplimiento de los compromisos adquiridos al firmar y ratificar convenios e instrumentos 
internacionales; el contar con datos serios, confiables y actualizados sobre la biodiversidad nacional es parte de estos compromisos que se encadenan con muchos otros.

El reto a mediano plazo es abarcar también el estado de conservación de especies y la integración de información sobre ecosistemas (conocimiento y estado), esfuerzos del país para la conservación de la biodiversidad (con énfasis en áreas silvestre protegidas), así como la sistematización de información de especies contenida en las diferentes colecciones del país.

\section{Agradecimientos}

Agradecemos a la Escuela de Ciencias Biológicas por creer en la importancia de los datos sobre la biodiversidad y apoyar la reactivación de esta iniciativa. Al SINAC y CONAGEBIO por la confianza depositada para que la UNA desarrolle el proceso de sistematización y contribuir así en el fortalecimiento de alianzas institucionales. Al Museo Nacional por permitir acceder a su valiosa base de datos. Un especial agradecimiento a todos y todas los expertos y expertas que colaboraron con sus conocimientos para actualizar datos y contribuir en la conservación de la biodiversidad.

\section{Referencias}

Avalos, G. (2018). Still searching the rich coast: Biodiversity of Costa Rica, numbers, processes, patterns, and challenges. Chapter 7 in Pullaiah, T. (editor). (2019) Global Biodiversity, Volume 4, selected countries in the Americas and Australia. Waretown, NJ: Apple Academic Press, 2019. 4. CRC Press, USA. https://www.nhbs.com/global-biodiversity-volume-4-book, https://fieldstudies.org/about/team/ gerardo-avalos/

Consejo Nacional de Rectores (2015). Programa Estado de la Nación. Capítulo Armonía con la Naturaleza. https://estadonacion.or.cr/informes/

Dean, H.K., Sibaja-Cordero, J.A. \& Cortés, J. (2010). Occurrence of the Phoronid Phoronopsis albomaculata in Cocos Island, Costa Rica. Pacific Science 64 (3): 459-462. https://doi.org/10.2984/64.3.459
Larsen, B. B.; Miller, E. C., Rhodes, M. K. \& Wiens J.J. (2017). Inordinate Fondness Multiplied and Redistributed: the Number of Species on Earth and the New Pie of Life. The Quarterly Review of Biology 92, No. 3: 229-265. https://doi.org/10.1086/693564

Ministerio de Recursos Naturales, Energía y Minas (MIRENEM), Museo Nacional, Instituto Nacional de Biodiversidad (INBio). (1992). Estudio Nacional de Biodiversidad. Costos, Beneficios y Necesidades de Financiamiento de la Conservación de la Diversidad Biológica en Costa Rica. PNUMA, Gobierno de Canadá (mimeografiado).

Mora. C.; Tittensor D.P., Adl. S., Simpson A.G.B, Worm. B. (2011). How Many Species Are There on Earth and in the Ocean? PLOS Biology 9(8): e1001127. https:// doi.org/10.1371/journal.pbio.1001127

Neuhaus, B. \& Blasche, T. (2006). Fissuroderes, a new genus of Kinorhyncha (Cyclorhagida) from the deep sea and continental shelf of New Zealand and from the continental shelf of Costa Rica. Zoologischer Anzeiger-A Journal of Comparative Zoology. Volume 245 (1): 19-52. https://doi.org/10.1016/j. jcz.2006.03.003.

Obando, V. (2002). Biodiversidad en Costa Rica. Estado del conocimiento y gestión. MINAE-INBio. Editorial INBio. $1^{\circ}$ Ed. Santo Domingo de Heredia, Costa Rica. 81 p.

Obando, V. (2007). Biodiversidad de Costa Rica en cifras. MINAE-SINAC-INBio. Editorial INBio. Santo Domingo de Heredia, Costa Rica. 21 p.

SINAC-MINAE, CONAGEBIO. (2018). VI Informe de País sobre el Cumplimiento del Convenio sobre Diversidad Biológica (CDB). https://chmcostarica.go.cr/recursos/documentos-y-publicaciones/vi-informe-de-costa-rica-al-convenio-de-diversidad-biologica

Vargas, J.A., Acuña-González, J., Vásquez, F. \& SibajaCordero, J.A. (2016). Brachiopods, sipunculans, enteropneusts and metals from two estuarine tidal flats, Pacific, Costa Rica. Rev. Biol. Trop. Vol. 64 (3): 1311-1331. https://revistas.ucr.ac.cr/index.php/rbt/ article/view/23251/25831

Werthman, I. \& Cortés, J. (2009). Marine Biodiversity of Costa Rica, Central America. Monographiae Biologicae. Volume 86. Series Editor H.J. Dumont Springer. springer. 544 p. 